\begin{tabular}{|c|c|c|c|c|c|c|}
\hline \multirow{4}{*}{ Impact Factor: } & ISRA (India) & $=3.117$ & SIS (USA) & $=0.912$ & ICV (Poland) & $=6.630$ \\
\hline & ISI (Dubai, UAE & $=\mathbf{0 . 8 2 9}$ & РИНЦ (Russia & $=0.156$ & PIF (India) & $=1.940$ \\
\hline & GIF (Australia) & $=0.564$ & ESJI (KZ) & $=5.015$ & IBI (India) & $=4.260$ \\
\hline & JIF & $=1.500$ & SJIF (Morocco & $=5.667$ & & \\
\hline
\end{tabular}

\begin{tabular}{|c|c|}
\hline $\begin{array}{l}\text { SOI: } 1.1 / 7 \\
\text { International S } \\
\text { Theoretical } \mathbf{\&}\end{array}$ & $\begin{array}{l}\frac{\mathrm{AS}}{\mathrm{DO}} \text { DO } 10.15863 / \mathrm{TAS} \\
\text { ientific Journal } \\
\text { pplied Science }\end{array}$ \\
\hline p-ISSN: 2308-4944 (print) & e-ISSN: 2409-0085 (online) \\
\hline Year: 2018 & Volume: 68 \\
\hline Published: 30.12 .2018 & tttp://T-Science.org \\
\hline
\end{tabular}

15.00.00 Psychology

UDC 618.177-089.888.11+159.9.07
QR - Issue

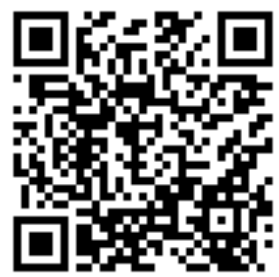

Assistant of the Department of Psychotherapy, the Psychology and Social Studies Faculty, Pirogov Russian National Research Medical University

Zh.R. Gardanova

Doctor of Medical Sciences, Professor, Head of the Department of Psychotherapy, the Psychology and Social Studies Faculty,

Pirogov Russian National Research Medical University

V.I. Esaulov Assistant of the Department of Psychotherapy, the Psychology and Social Studies Faculty, Pirogov Russian National Research Medical University

S.S. Silin

Clinical Psychologist, Pirogov Russian National Research Medical University

\title{
PSYCHOLOGICAL DIAGNOSTICS OF ANXIETY AND DEPRESSION LEVEL OF WOMEN OF OLDER REPRODUCTIVE AGE IN THE IN VITRO FERTILIZATION PROGRAM
}

Abstract: Psychological diagnostics of 75 women of older reproductive age was carried out. Its aim was to determine the level of anxiety and depression as well as to assess the personal profile of patients and their quality of life.

It is noted that the level of anxiety and depression of women of the older reproductive age in the in vitro fertilization program is higher than of healthy women.

Taking into account the level of depression and anxiety could help doctors and psychologists to promptly provide women undergoing IVF treatment with psychocorrection aid.

Key words: women, older reproductive age, anxiety, depression, infertility, in vitro fertilization, psychodiagnostics.

Language: Russian

Citation: Kalina, S. A., Gardanova, Z. R., Esaulov, V. I., \& Silin, S. S. (2018). Psychological diagnostics of anxiety and depression level of women of older reproductive age in the in vitro fertilization program. ISJ Theoretical \& Applied Science, 12 (68), 306-311.

Soi: http://s-o-i.org/1.1/TAS-12-68-45 Doi: crostef https://dx.doi.org/10.15863/TAS.2018.12.68.45

\section{ПСИХОЛОГИЧЕСКАЯ ДИАГНОСТИКА ТРЕВОЖНО-ДЕПРЕССИВНЫХ СОСТОЯНИЙ ЖЕНЩИН СТАРШЕГО РЕПРОДУКТИВНОГО ВОЗРАСТА В ПРОГРАММЕ ЭКСТРАКОРПОРАЛЬНОГО ОПЛОДОТВОРЕНИЯ}

Аннотация: Проведена психологическая диагностика у 75 женщин старшего репродуктивного возраста, направленная на исследование уровня тревоги и депрессии, оценку личностного профиля пациенток и качества жизни.

Отмечено, что у женщин старшей репродуктивной группь в программе экстракорпорального оплодотворения уровень депрессии и тревоги выше, чем у здоровых женщин. 


\begin{tabular}{|c|c|c|c|c|c|c|}
\hline \multirow{4}{*}{ Impact Factor: } & ISRA (India) & $=3.117$ & SIS (USA) & $=0.912$ & ICV (Poland) & $=6.630$ \\
\hline & ISI (Dubai, UAF & $=0.829$ & РИНЦ (Russia & $=0.156$ & PIF (India) & $=1.940$ \\
\hline & GIF (Australia) & $=0.564$ & ESJI (KZ) & $=5.015$ & IBI (India) & $=4.260$ \\
\hline & JIF & $=1.500$ & SJIF (Morocco & $=5.667$ & & \\
\hline
\end{tabular}

Учет уровня тревожности и депрессии могут помочь врачам и психологам своевременно оказать психокоррекционную помощь женщиннам, проходящим лечение по программе ЭКО.

Ключевые слова: женщины, старший репродуктивный возраст, тревога, депрессия, бесплодие, искусственное оплодотворение, психодиагностика.

\section{Введение.}

Бесплодие женщин существенно влияет на психоэмоциональный статус, вызывая состояние хронического стресса и разнообразные расстройства эмоциональной сферы.

В настоящее время численность населения Российской Федерации вновь начала снижаться, остро обнажив проблему демографического кризиса.

В этой связи лечение бесплодия имеет особую актуальность в ряду мер, направленных на повышение рождаемости. Перспективным способом лечения бесплодия является метод экстракорпорального оплодотворения (ЭКО). Специалистами выявлена тенденция к увеличению числа женщин, прибегающих к программе ЭКО в старшем детородном возрасте [7; 10, с.48-53].

В категории женщин старшей возрастной группы накоплен опыт переживаний и комплексов по поводу собственного бесплодия, обрастающий разочарованиями, неверием, отчаянием на фоне менее гибкой личностной структуры [8].

Подобные состояния мало изучены у женщин старшего фертильного возраста.

Поэтому актуальным является оценка особенностей личностного реагирования женщин на собственное бесплодие, которое можно получить с помощью психодиагностики.

Это дает возможность ввести в терапию помимо общего бережного и деликатного подхода к переживаниям пациенток еще и специфические личностно-ориентированные тактики, позволяющие повысить эффективность проводимой терапии.

Цель исследования: выявить и определить уровень тревожных расстройств у женщин старшего репродуктивного возраста в программе ЭКО.

\section{Характеристика выборки и методы исследования: \\ Всего в исследовании приняли участие 75} женщин старшего репродуктивного возраста $(44.5 \pm 7.5$ года) проходящие лечение в Национальном медицинском исследовательском центре акушерства, гинекологии и перинатологии Министерства здравоохранения РФ:

- 25 женщин составили контрольную группу (группа 2), в нее вошли женщины с благоприятной беременностью
- 25 женщин с бесплодием вошли в экспериментальную группу (группа 1)

- 25 женщин с бесплодием и донорской клеткой вошли в еще одну экспериментальную группу (группа 3).

Был использован набор психологических тестов: Шкала депрессии Бека; Шкала оценки уровня реактивной и личностной тревожности Спилбергера - Ханина; Личностный опросник Шмишека; Неспецифический опросник для изучения качества жизни; Торонтская шкала алексетимии; Копинг-тест Лазаруса.

\section{Результаты исследования и их} обсуждение.

В результате нашего исследования были изучены различные аспекты психоэмоционального состояния у группы женщин старшего репродуктивного возраста в программе ЭКО (группа 1), контрольной группы здоровых женщин (группа 2) и группы женщин старшего репродуктивного возраста с бесплодием, в программе которых используется донорская клетка (группа 3).

По данным Торонтской шкалы алекситимии установлено, что женщины обеих экспериментальных групп (группы 1, а также группы 3) чаще оказываются в группе риска алекситимии, в отличие от женщин контрольной группы.

Вероятно, это связано с тем, что женщины старшего репродуктивного возраста в программе ЭКО испытывают сильный стресс, так как находятся в непривычных условиях зависимости от препаратов, процедур под постоянным контролем врачей [9]. Можно констатировать тот факт, что на основе вышеперечисленного, они попадают в группу риска алекситимии.

Различие между группой 1 и группой 3 не имеет статистической значимости.

Средние показатели данных по опроснику Шмишека показывают, что женщинам с бесплодием (группа 1) свойственно иметь взрывной экзальтированный характер, женщинам с бесплодием и донорской клеткой характерна тревожность (группа 3), а женщинам контрольной группы присуща гипертимность(группа 2) [2, с.429].

На основе полученных результатов шкалы депрессии Бека выявлено, что женщины с бесплодием без донорской клетки в программе ЭКО (группа 1) имеют легкие и средние депрессивные состояния, в отличие от женщин контрольной группы $[11 ; 16$, с.27-34]. 


\begin{tabular}{|c|c|c|c|c|c|c|}
\hline \multirow{4}{*}{ Impact Factor: } & ISRA (India) & $=3.117$ & SIS (USA) & $=0.912$ & ICV (Poland) & $=6.630$ \\
\hline & ISI (Dubai, UAF & $=0.829$ & РИНЦ (Russia) & $=0.156$ & PIF (India) & $=1.940$ \\
\hline & GIF (Australia) & $=0.564$ & ESJI (KZ) & $=\mathbf{5 . 0 1 5}$ & IBI (India) & $=4.260$ \\
\hline & JIF & $=1.500$ & SJIF (Morocco) & $=5.667$ & & \\
\hline
\end{tabular}

У женщин с бесплодием и донорской клеткой в программе ЭКО (группа 3) чаще встречаются средние и выраженные депрессивные состояния, и лишь в единичных случаях депрессия $[11 ; 16$, с.27-34]. Различие между 1 и 3 группами женщин с бесплодием статистически значимое.

Показатели шкал депрессии Бека по трем группам приведены на Рис.1

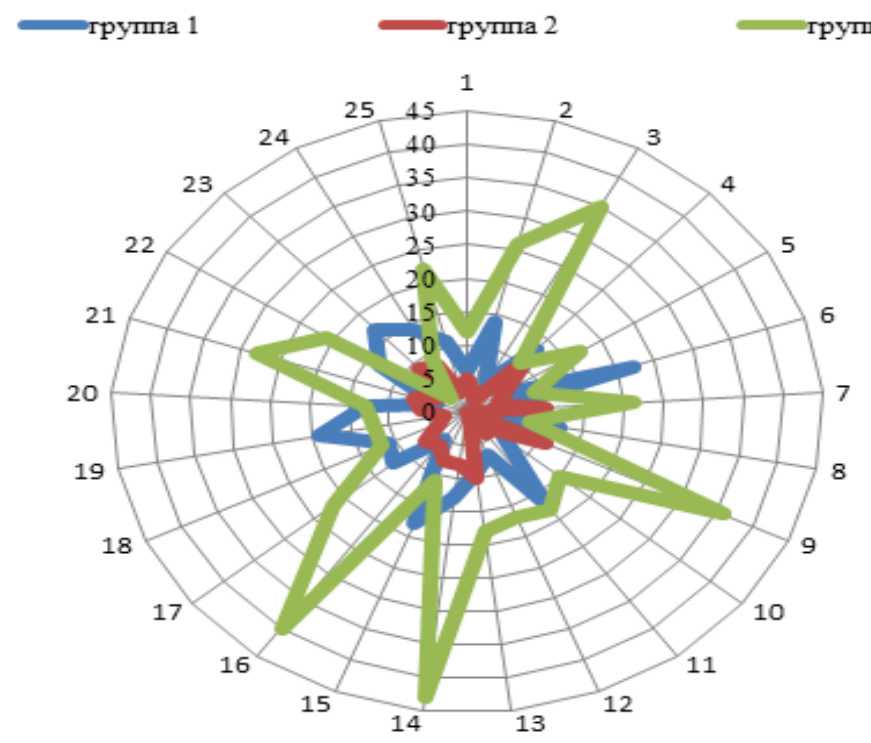

Рис.1 Показатели шкалы депрессии Бека у женщин 1, 2 и 3 группы.

Помимо этого, испытуемым был предложен опросник «Способы совладающего поведения» по Лазарусу.

В группе женщин старшего репродуктивного возраста в программе ЭКО (группа 1) было выявлено, что копинг- стратегии по типу самоконтроля находятся в области высокой напряженности. Стратегия самоконтроля предполагает варианты преодоления негативных переживаний путем сдерживания эмоций, минимизации их влияния на оценку ситуации. У личности может наблюдаться стремление скрывать от окружающих свои переживания и побуждения в связи с проблемной ситуацией. Выбор стратегии поведения будет склоняться к сверхконтролю и стремлению к самообладанию. Часто такое поведение свидетельствует о боязни самораскрытия, чрезмерной требовательности к себе.

В группе женщин с бесплодием и донорской клеткой в программе ЭКО (группа 3) отмечена копинг-стратегия по типу поиска социальной поддержки, находящаяся в зоне риска дезадаптации. Женщины, оказавшись в сложной тревожной ситуации, стремятся разделить свои переживания, хотят быть выслушанными. В данном случае возникает риск формирования зависимого поведения или чрезмерного ожидания от врачей и процедур.
Исследование качества жизни беременных женщин по опроснику SF-36 не показало статистически значимых различий.

Между тем, такие показатели здоровья, как физическое функционирование, ролевая деятельность, телесная боль, общее здоровье, жизнеспособность, социальное функционирование, эмоциональное состояние и психическое здоровье у женщин контрольной группы выше, чем у женщин с бесплодием в программе ЭКО с донорской клеткой и без [14; 15, c.876-886].

Возможно, отсутствие статистически значимых отличий связано с высокими достижениями в сфере карьерного роста, так как большинство испытуемых женщин добились успехов в собственной профессиональной области.

При изучении уровня личностной тревожности с помощью теста СпилбергераХанина не выявлено статистически значимых различий при сравнении показателей группы женщин с бесплодием (группа 1) и женщин контрольной группы (группа 2).

В единичных случаях у женщин старшего репродуктивного возраста с донорской клеткой в программе ЭКО (группа 3) была отмечена высокая личностная тревожность [4, с.85-92].

Показатели личностной тревожности по тесту Спилбергера-Ханина приведены на Рис.2: 


\begin{tabular}{llllll} 
& ISRA (India) $=\mathbf{3 . 1 1 7}$ & SIS (USA) $=\mathbf{0 . 9 1 2}$ & ICV (Poland) & $\mathbf{= 6 . 6 3 0}$ \\
Impact Factor: & ISI (Dubai, UAE) $=\mathbf{0 . 8 2 9}$ & PUHL (Russia) $=\mathbf{0 . 1 5 6}$ & PIF (India) & $=\mathbf{1 . 9 4 0}$ \\
& GIF (Australia) $=\mathbf{0 . 5 6 4}$ & ESJI (KZ) & $\mathbf{5 . 0 1 5}$ & IBI (India) & $=\mathbf{4 . 2 6 0}$ \\
& JIF & $\mathbf{1 . 5 0 0}$ & SJIF (Morocco) $=\mathbf{5 . 6 6 7}$ & & \\
\hline
\end{tabular}

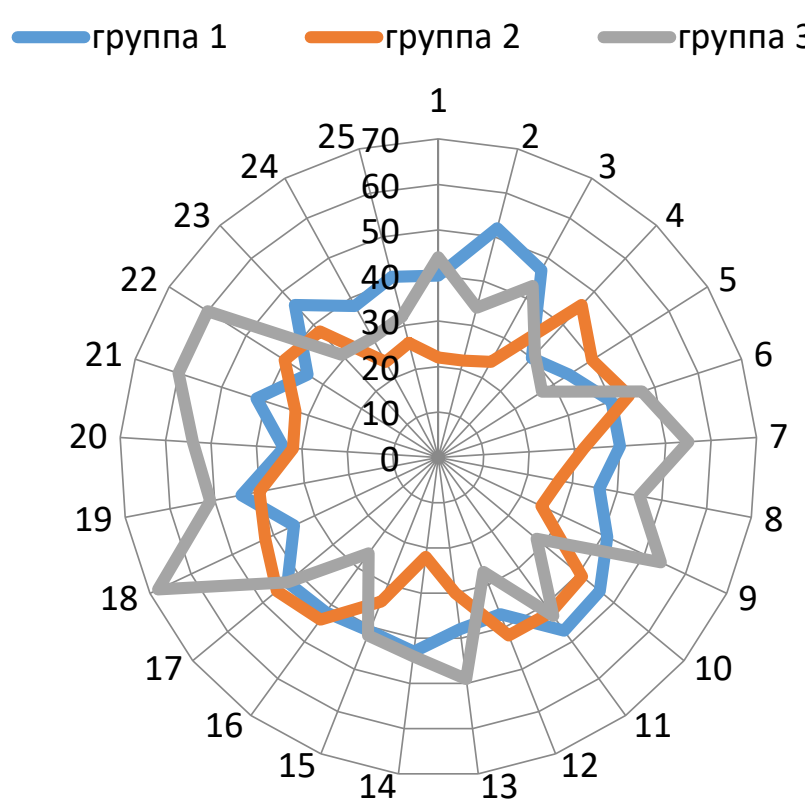

Рис. 2. Показатели личностной тревожности по тесту Спилбергера-Ханина у женщин 1, 2 и 3 группы.

При исследовании тревожности с помощью теста Спилбергера-Ханина ситуационная тревожность у женщин с бесплодием (группа1) статистически значительно выше, чем у женщин контрольной группы(группа 2). Женщины с бесплодием и донорской клеткой (группа 3) также значительно тревожнее женщин контрольной группы (группа 2). Это можно объяснить реакцией на сложившуюся ситуацию бесплодия, беспокойством перед проведением процедур, страхом за плод и собственное здоровье [12]. Различия между группой 1 и группой 3 находятся в зоне незначимости.

Показатели ситуационной тревожности по тесту Спилбергера-Ханина приведены в Рис.3.

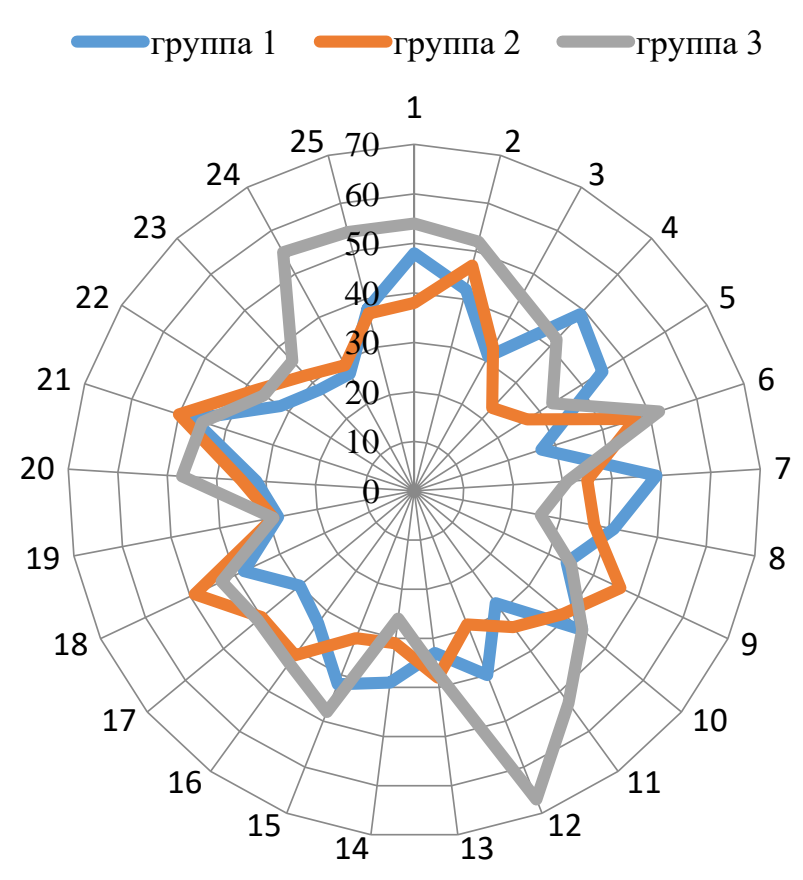

Рис. 3. Показатели ситуационной тревожности по тесту Спилбергера-Ханина у женщин 1, 2 и 3 группы. 


\begin{tabular}{|c|c|c|c|c|c|c|}
\hline \multirow{4}{*}{ Impact Factor: } & ISRA (India) & $=3.117$ & SIS (USA) & $=0.912$ & ICV (Poland) & $=6.630$ \\
\hline & ISI (Dubai, UAE & $=0.829$ & РИНЦ (Russia) & $=0.156$ & PIF (India) & $=1.940$ \\
\hline & GIF (Australia) & $=0.564$ & ESJI (KZ) & $=5.015$ & IBI (India) & $=4.260$ \\
\hline & JIF & $=1.500$ & SJIF (Morocco) & $=5.667$ & & \\
\hline
\end{tabular}

Резюмируя полученные результаты, можно сделать следующие выводы:

1) Большинство женщин старшего репродуктивного возраста в программе ЭКО (с донорской клеткой и без) попадают в группу риска алекситимии.

2) Женщины с бесплодием в программе ЭКО имеют легкие и средние депрессивные состояния, в отличие от женщин контрольной группы.

У женщин с бесплодием и донорской клеткой в программе ЭКО чаще встречаются средние и выраженные депрессивные состояния.

3) Ситуационная тревожность у женщин с бесплодием в программе ЭКО (с донорской клеткой и без) находится на высоком уровне.

4) Уровень качества жизни во всех группах не имеет статистических различий.

5) Большинство женщин старшего репродуктивного возраста в программе ЭКО с донорской клеткой имеют тревожный характер, большинство женщин с бесплодием и без донорской клетки в программе ЭКО имеют взрывной экзальтированный тип характера.

6) Женщины с бесплодием и донорской клеткой в программе ЭКО находятся в зоне риска дезадаптации, используя копинг-стратегию по типу поиска социальной поддержки. Женщины с бесплодием и без донорской клетки в программе ЭКО склонны к дезадаптивному самоконтролю.

\section{Заключение.}

При исследовании психоэмоциональных расстройств женщин старшей репродуктивной группы в программе ЭКО выявлено, что большинство женщин старшего детородного о возраста в программе ЭКО (с донорской клеткой и без) попадают в группу риска алекситимии. Значимых различий между группой женщин с донорской клеткой и группой женщин с бесплодием в программе ЭКО без донорской клетки нет.

Обследованные женщины с бесплодием в программе ЭКО имели легкие и средние депрессивные состояния, в отличие от женщин контрольной группы. Ситуационная тревожность у женщин с бесплодием в программе ЭКО (с донорской клеткой и без) находится на высоком уровне.

Женщины с бесплодием и донорской клеткой в программе ЭКО находятся в зоне риска дезадаптации, используя копинг-стратегию по типу поиска социальной поддержки, тогда, как женщины с бесплодием и без донорской клетки в программе ЭКО склонны к дезадаптивному самоконтролю.

Четкая диагностика психоэмоционального состояния позволяет использовать дифференцированный подход к ведению этой группы пациенток.

\section{References:}

1. Budik, A. M., \& Korabelnikova, E. A. (2009). Trevoga kak kliniko-psikhofiziologicheskiy fenomen. Obozreniye psikhiatrii i meditsinskoy psikhologii, № 4, 73-75.

2. Garanyan, N. G. (2010). Perfektsionizm $i$ vrazhdebnost kak lichnostnyye faktory depressivnykh $i$ trevozhnykh rasstroystv. dissertatsiya ... doktora psikhologicheskikh nauk: 19.00.04. (p.429). Moskva.

3. Gardanova, Z. R. (2008). Pogranichnyye psikhicheskiye rasstroystva $u$ zhenshchin $s$ besplodiyem $v$ programme vspomogatelnykh reproduktivnykh tekhnologiy $i$ ikh psikhoterapevticheskaya korrektsiya. diss. dokt. medits. nauk. (p.210). Moskva.

4. Gardanova, Z. R. (2007). Vliyaniye psikhicheskogo i psikhologicheskogo sostoyaniya na protsess provedeniya lecheniya besplodiya metodom ekstrakorporalnogo oplodotvoreniya. meditsinskiy zhurnal, №2, 85-92.

5. Zakharova, E. I. (2011). Kurs lektsiy «Psikhologicheskoye soprovozhdeniye beremennosti. MGU im. M.V. Lomonosova.

6. Zakharova, E. I. (2008). Razvitiye lichnosti v khode osvoyeniya roditelskoy pozitsii. Kulturno-istoricheskaya psikhologiya, № 2, 24-29.

7. Nazarenko,T. A. (2005). Znacheniye otsenki ovarialnogo rezerva $\mathrm{v}$ lechenii besplodiya $\mathrm{u}$ zhenshchin starshego reproduktivnogo vozrasta. Problemy reproduktsii [Elektronnyy resurs], № 2.

http://www.mediasphera.aha.ru/journais/reprod uction

8. Karibayeva, S. K. (2009). Otsenka sostoyaniya $i$ nauchnoye obosnovaniye klinikoorganizatsionnykh form povysheniya kachestva spetsializirovannoy pomoshchi zhenshchinam. 


\begin{tabular}{|c|c|c|c|c|c|c|}
\hline \multirow{4}{*}{ Impact Factor: } & ISRA (India) & $=3.117$ & SIS (USA) & $=0.912$ & ICV (Poland) & $=6.630$ \\
\hline & ISI (Dubai, UAE & $=0.829$ & РИНЦ (Russia) & $=0.156$ & PIF (India) & $=1.940$ \\
\hline & GIF (Australia) & $=0.564$ & ESJI (KZ) & $=\mathbf{5 . 0 1 5}$ & IBI (India) & $=4.260$ \\
\hline & JIF & $=1.500$ & SJIF (Morocco) & $=5.667$ & & \\
\hline
\end{tabular}

stradayushchim besplodiyem. $v$ usloviyakh tsentra EKO. avtoreferat dis. ... kandidata meditsinskikh nauk : 14.00.33. 14.00.01. Sankt-Peterburg.

9. Kokh, L. I., \& Egorkina, Y. V. (2008). Vliyaniye trevozhnosti na techeniye beremennosti i rodov $\mathrm{u}$ zhenshchin kraynikh vozrastnykh grupp. SMZh, №1-2.

10. Kristich, E. V., Krasnopolskaya, K. V., \& Kabanova, D. I. (2010). Novyye podkhody k povysheniyu effektivnosti EKO u zhenshchin starshego reproduktivnogo vozrasta. Akusherstvo i ginekologiya, № 2, 48-53.

11. Tyutyunnik, V. L., Mikhaylova, O. I., \& Chukhareva, N. A. (2012). Psikhoemotsionalnyye rasstroystva pri beremennosti. Neobkhodimost ikh korrektsii. Russkiy med. Zhurn., №5.

12. Filippova, G. G. (2011). Psikhologiya reproduktivnoy sfery cheloveka: metodologiya. teoriya. Praktika. Meditsinskaya psikhologiya $v$ Rossii, № 6.

13. Khritinin, D. F., Gardanova, Z. R., \& Kulakova, E. V. (2007). Organizatsiya psikhoterapevticheskoy pomoshchi zhenshchinam. nuzhdayushchimsya v surrogatnom materinstve $\mathrm{V}$ programme ekstrakorporalnogo oplodotvoreniya. Kazan. Zh.Obshchestvennoye zdorovye $i$ zdravookhraneniya, № 4, 87-90.

14. Aarts, J. W., et al. (2011, March 3). Relationship between quality of life and distress in infertility: a validation study of the Dutch FertiQoL. Hum. Reprod, 26 (5), 1112-1118. Epub.

15. Kraaij, V., et al. (2010, May 7). Cognitive coping, goal adjustment, and depressive and anxiety symptoms in people undergoing infertility treatment: a prospective study. Hlth Psychol., 15 (6), 876-886. Epub.

16. Volgsten, H., Ekselius, L., Poromaa, I. S., \& Svanberg, A. S. (2010). Personality traits associated with depressive and anxiety disorders in infertile women and men undergoing in vitro fertilization treatment. Acta Obstet. Gynecol. Scand., 89 (1), 27-34. 